\title{
PRIMITIVE ELEMENTS IN FREE GROUPS
}

\author{
MARTIN J. EVANS
}

(Communicated by Bhama Srinivasan)

\begin{abstract}
Let $F_{n}$ denote the free group of rank $n$ generated by $x_{1}, x_{2}, \ldots, x_{n}$ We say that $y \in F_{n}$ is a primitive element of $F_{n}$ if it is contained in a set of free generators of $F_{n}$. In this note we construct, for each integer $n \geq 4$, an $(n-1)$-generator group $H$ that has an $n$-generator, 2-relator presentation $H=\left\langle x_{1}, \ldots, x_{n} \mid r_{1}, r_{2}\right\rangle$ such that the normal closure of $\left\{r_{1}, r_{2}\right\}$ in $F_{n}$ does not contain a primitive element of $F_{n}$.
\end{abstract}

\section{INTRODUCTION}

Let $F_{n}$ denote the free group of rank $n$, freely generated by $x_{1}, x_{2}, \ldots, x_{n}$. We say that $x \in F_{n}$ is a primitive element of $F_{n}$ if it is contained in a set of free generators of $F_{n}$.

According to [2, p. 92], F. Waldhausen asked "If $G=\left\langle x_{1}, x_{2}, \ldots, x_{n}\right| r_{1}$, $\left.\ldots, r_{m}\right\rangle$ can be generated by fewer than $n$ elements, then does the normal closure of $\left\{r_{1}, \ldots, r_{m}\right\}$ in $F_{n}$ contain a primitive element of $F_{n}$ ?" A negative answer has been obtained by G. A. Noskov [5]. His ingenious argument is partly geometric and uses the "Hairy Ball" theorem at a crucial point.

The purpose of this note is to prove the following.

Theorem. For each integer $n \geq 4$, there exists an $(n-1)$-generator group $H$ that has an n-generator, 2-relator presentation $H=\left\langle x_{1}, \ldots, x_{n} \mid r_{1}, r_{2}\right\rangle$ such that the normal closure of $\left\{r_{1}, r_{2}\right\}$ in $F_{n}$ does not contain a primitive element of $F_{n}$.

We remark that any presentation that provides a negative answer to Waldhausen's question has at least two relators. For suppose that $G=\left\langle x_{1}, \ldots, x_{n} \mid r\right\rangle$ can be generated by fewer than $n$ elements. It follows easily from [3, Corollary 5.14.2] that $G$ is free and we can now use a result of J. H. C. Whitehead (see [2, Chapter 2], Proposition 5.10) to deduce that $r$ is a primitive element of $F_{n}$.

Received by the editors May 15, 1987, and, in revised form, June, 1988.

1980 Mathematics Subject Classification (1985 Revision). Primary 20F05; Secondary 20E05. 


\section{The MOdUles $M$ AND $B$}

Let $G=\left\langle x, y \mid x^{2}=y^{3}\right\rangle$; the trefoil group. M. J. Dunwoody [1] has shown that $M=\mathbf{Z} G \oplus \mathbf{Z} G$, the free right $G$-module of rank 2, can be decomposed as $M=C \oplus B$ where $C \cong \mathbf{Z} G$ and $B$ cannot be generated (as a $G$-module) by a single element. With this notation we have

Lemma 1. $M$ cannot be generated (as a G-module) by two elements, one of which lies in $B$.

Proof. It is convenient to view $M$ as an external direct sum of $\mathrm{Z} G$ and $B$. Thus $M=\{(z, b) \mid z \in \mathbf{Z} G, b \in B\}$.

Suppose, for a contradiction, that $M$ is generated by $(z, b)$ and $\left(0, b^{\prime}\right)$. Note that $b$ and $b^{\prime}$ together generate $B$. Now there exist $\alpha, \beta \in \mathbf{Z} G$ such that $(z, b) \alpha+\left(0, b^{\prime}\right) \beta=(1,0)$. So $z \alpha=1$ and $b \alpha=-b^{\prime} \beta$. Since $z \alpha=1$, a result of Kaplansky (see [4]) implies that $\alpha z=1$. Therefore $b=-b^{\prime} \beta z$ and $B$ is generated by $b^{\prime}$. However, by the preceding remarks, $B$ cannot be generated by a single element and we have obtained the desired contradiction.

Since $M=\mathbf{Z} G \oplus \mathbf{Z} G, M$ can be generated by two elements, $m_{1}$ and $m_{2}$, say. Furthermore, $B$ is an image of $M$ so $B$ can be generated by two elements, $b_{1}$ and $b_{2}$. Also, $C \cong \mathbf{Z} G$, so $C$ can be generated by a single element $c$. Note that $M$ is generated by $c, b_{1}$ and $b_{2}$. We fix this notation once and for all.

\section{THE GROUPS $W$ AND $\bar{W}$}

From now on, $n$ denotes a fixed integer with $n \geq 4$. Let $F$ denote the free group of rank $n-2$, generated by $y_{1}, y_{2}, \ldots, y_{n-2}$, and let $\theta: F \rightarrow G$ be any epimorphism.

Define an action of $F$ on $M$ by $m^{f}=m^{f \theta}$, for all $m \in M$ and $f \in F$. Note that $K \subseteq M$ is an $F$-submodule of $M$ if and only if it is a $G$-submodule of $M$, so we may write unambiguously of submodules of $M$. Combining this remark with Lemma 1 yields

Lemma 2. $M$ cannot be generated as an $F$-module by two elements, one of which lies in $B$.

Since $M$ is an $F$-module we can form $W=M \rtimes F$, the semidirect product of $M$ and $F$. We shall identify $M$ and $F$ with their natural copies in $W$ so that $W=M F$ and $M \cap F$ is trivial.

Recall that the elements $m_{1}$ and $m_{2}$ generate $M$, so $W=\left\langle y_{1}, \ldots, y_{n-2}\right.$, $\left.m_{1}, m_{2}\right\rangle$ and $W$ is $n$-generator.

Lemma 3. $W$ cannot be generated by $n$ elements, one of which lies in $B$.

Proof. Let $A$ be any group. The following transformations of the set of $s$-tuples $\left(a_{1}, a_{2}, \ldots, a_{s}\right)$, where $a_{1}, a_{2}, \ldots, a_{s} \in A$, are called elementary Nielsen transformations: (i) permuting the $a_{i}$ 's (ii) inverting $a_{i}$ for some $i$ (iii) leaving $a_{i}$ 
fixed for $i \neq j$ and replacing $a_{j}$ with $a_{k}^{ \pm 1} a_{j}$ or $a_{j} a_{k}^{ \pm 1}$ for some $k \neq j . \mathrm{Ob}$ serve that if $\sigma$ is an elementary Nielsen transformation and $\left(a_{1}, a_{2}, \ldots, a_{s}\right) \sigma=$ $\left(a_{1}^{\prime}, a_{2}^{\prime}, \ldots, a_{s}^{\prime}\right)$ then $\left\langle a_{1}, a_{2}, \ldots, a_{s}\right\rangle=\left\langle a_{1}^{\prime}, a_{2}^{\prime}, \ldots, a_{s}^{\prime}\right\rangle$.

Now suppose that the lemma is false so that $W=\left\langle h_{1} f_{1}, h_{2} f_{2}, \ldots, h_{n-1} f_{n-1}, b\right\rangle$ where $h_{1}, \ldots, h_{n-1} \in M, f_{1}, \ldots, f_{n-1} \in F$ and $b \in B$. Clearly $\left\langle f_{1}, f_{2}, \ldots, f_{n-1}\right\rangle=F$. It is well known (e.g. [3, Chapter 3]) that there exists a finite sequence of elementary Nielsen transformations $\sigma_{1}, \sigma_{2}, \ldots, \sigma_{l}$ such that $\left(f_{1}, f_{2}, \ldots, f_{n-1}\right) \sigma_{1} \sigma_{2} \cdots \sigma_{l}=\left(y_{1}, y_{2}, \ldots, y_{n-2}, 1\right)$. Apply the same transformation to $\left(h_{1} f_{1}, h_{2} f_{2}, \ldots, h_{n-1} f_{n-1}\right)$, we obtain

$$
\left(h_{1} f_{1}, h_{2} f_{2}, \ldots, h_{n-1} f_{n-1}\right) \sigma_{1} \sigma_{2} \cdots \sigma_{l}=\left(k_{1} y_{1}, k_{2} y_{2}, \ldots, k_{n-1} y_{n-2}, k_{n-1}\right)
$$

for some $k_{1}, k_{2}, \ldots, k_{n-1} \in M$. It follows that $\left\langle h_{1} f_{1}, h_{2} f_{2}, \ldots, h_{n-1} f_{n-1}\right\rangle=$ $\left\langle k_{1} y_{1}, k_{2} y_{2}, \ldots, k_{n-2} y_{n-2}, k_{n-1}\right\rangle$ so that

$$
W=\left\langle k_{1} y_{1}, k_{2} y_{2}, \ldots, k_{n-2} y_{n-2}, k_{n-1}, b\right\rangle \text {. }
$$

Let $K$ denote the submodule of $M$ generated by $k_{1}, \ldots, k_{n-2}$ and let $S=$ $\left\langle k_{1} y_{1}, \ldots, k_{n-2} y_{n-2}\right\rangle$. Now $S /(K \cap S) \cong K S / K=K F / K \cong F /(F \cap K) \cong F$ so $S$ has a free group of rank $n-2$ as a homomorphic image. However, $S$ can be generated by $n-2$ elements and it follows that $S$ is free of rank $n-2$.

Now let $N$ denote the submodule of $M$ generated by $k_{n-1}$ and $b$. We have $W=N S$ so that $M=M \cap N S=N(M \cap S)$. Since $S$ is free of rank $n-2$ it has no nontrivial abelian normal subgroups, so $M \cap S$ is trivial and we deduce that $N=M$. Thus $M$ is generated by two elements $k_{n-1}$ and $b$, one of which lies in $B$. This contradicts Lemma 2 and completes the proof.

Now $B$ is a submodule of $M$ so $B \triangleleft W$. Define $\bar{W}=W / B$ and write $\bar{w}$ for the image of $w \in W$ under the natural homomorphism $\pi: W \rightarrow W / B$. Since $M$ is generated by $c, b_{1}$ and $b_{2}$, we have $W=\left\langle y_{1}, \ldots, y_{n-2}, c, b_{1}, b_{2}\right\rangle$. Therefore $\bar{W}=\left\langle\bar{y}_{1}, \ldots, \bar{y}_{n-2}, \bar{c}\right\rangle$ and $\bar{W}$ is $(n-1)$-generator.

In light of Lemma 4 below, it is interesting to observe that $\bar{W}$ is isomorphic to the semi-direct product of $\mathrm{Z} G$ and $F$, where $F$ acts on $\mathrm{Z} G$ via $\theta$.

Our final lemma also established some notation that will be used in the proof of the theorem.

Lemma 4. Let

$$
\begin{aligned}
& \eta: F_{n} \rightarrow W \text { be the epimorphism defined by } \\
& \eta: x_{i} \rightarrow y_{i} \text { for } i=1,2, \ldots, n-2, \\
& \eta: x_{n-1} \rightarrow m_{1}, \\
& \eta: x_{n} \rightarrow m_{2} .
\end{aligned}
$$

Furthermore, let $\pi$ denote the natural homomorphism $\pi: W \rightarrow W / B=\bar{W}$ and define an epimorphism $\phi: F_{n} \rightarrow \bar{W}$ by $\phi=\eta \pi$ so that

$$
\begin{aligned}
& \phi: x_{i} \rightarrow \bar{y}_{i} \quad i=1,2, \ldots, n-2, \\
& \phi: x_{n-1} \rightarrow \bar{m}_{1}, \\
& \phi: x_{n} \rightarrow \bar{m}_{2} .
\end{aligned}
$$


Then $\operatorname{ker} \phi$ does not contain a primitive element of $F_{n}$.

Proof. Suppose $p \in \operatorname{ker} \phi$ is a primitive element of $F_{n}$. Then there exist $p_{1}, p_{2}, \ldots, p_{n-1} \in F_{n}$ such that $\left\langle p_{1}, p_{2}, \ldots, p_{n-1}, p\right\rangle=F_{n}$. Now $W=$ $\left\langle p_{1} \eta, p_{2} \eta, \ldots, p_{n-1} \eta, p \eta\right\rangle$ where $p \eta \pi=p \phi=1$ so that $p \eta \in \operatorname{ker} \pi=B$. Thus $W$ can be generated by $n$ elements, one of which lies in $B$. This contradicts Lemma 3 and the result follows.

We point out that the significance of Lemma 4 is that the $(n-1)$-generator group $\bar{W}$ can be written as $\bar{W}=F_{n} / \operatorname{ker} \phi$, where $\operatorname{ker} \phi$ does not contain a primitive element of $F_{n}$.

\section{Proof OF THE THEOREM}

Recall that $\bar{W}=\left\langle\bar{y}_{1}, \bar{y}_{2}, \ldots, \bar{y}_{n-2}, \bar{c}\right\rangle$ and let $z$ be a preimage of $\bar{c}$ under $\phi$.

Consider $L=\left\langle x_{1}, x_{2}, \ldots, x_{n-2}, z\right\rangle$, a subgroup of $F_{n}$. Let $\phi_{L}$ denote the restriction of $\phi$ to $L$ and note that $\phi_{L}: L \rightarrow \bar{W}$ is onto since $x_{i} \phi_{L}=\bar{y}_{i}$ for $i=1,2, \ldots, n-2$ and $z \phi_{L}=\bar{c}$. Therefore, there exist $z_{1}, z_{2} \in L$ such that $z_{1} \phi_{L}=\bar{m}_{1}$ and $z_{2} \phi_{L}=\bar{m}_{2}$.

We shall show that $H=\left\langle x_{1}, \ldots, x_{n} \mid x_{n-1} z_{1}^{-1}, x_{n} z_{2}^{-1}\right\rangle$ has the properties referred to in the statement of the theorem.

Let $R$ denote the normal closure of $\left\{x_{n-1} z_{1}^{-1}, x_{n} z_{2}^{-1}\right\}$ in $F_{n}$ so that $H \cong$ $F_{n} / R$. Firstly, $x_{n-1} R=z_{1} R$ and $x_{n} R=z_{2} R$ where $z_{1}, z_{2} \in\left\langle x_{1}, x_{2}, \ldots\right.$, $\left.x_{n-2}, z\right\rangle$ so that $F_{n} / R$ is generated by $x_{1} R, x_{2} R, \ldots, x_{n-2} R$ and $z R$. Therefore $H$ is $(n-1)$-generator. Moreover, $z_{1} \phi=\bar{m}_{1}=x_{n-1} \phi$ and $z_{2} \phi=\bar{m}_{2}=$ $x_{n} \phi$ so $x_{n-1} z_{1}^{-1}, x_{n} z_{2}^{-1} \in \operatorname{ker} \phi$. Therefore $R \leq \operatorname{ker} \phi$ and it follows immediately from Lemma 4 that $R$ does not contain any primitive elements of $F_{n}$. The theorem is proved.

\section{REFERENCES}

1. M. J. Dunwoody, Relation modules, Bull. London Math. Soc. 4 (1972), 151-155.

2. R. C. Lyndon and P. E. Schupp, Combinatorial group theory, Springer-Verlag, Berlin-Heidelberg-New York, 1977.

3. W. Magnus, A. Karrass and D. Solitar, Combinatorial group theory, Interscience, New York, 1966.

4. M. S. Montgomery, Left and right inverses in group algebras, Bull. Amer. Math. Soc. 75 (1969), 539-540.

5. G. A. Noskov, Primitive elements in a free group, Mat. Zametki 30(4) (1981), 497-500.

Department of Mathematics, Auburn University, Auburn, Alabama 36849

Current address: Department of Mathematics, University of Wisconsin-Parkside, Kenosha, Wisconsin 53141-2000 